\title{
Enhanced Data Generated With Electrons (EDGE) Special Issue Introduction
}

Continuing the series of workshops on electron energy-loss spectroscopy (EELS) started at Lake Tahoe in California 1990, and followed by meetings in Leukerbad, Switzerland (EELSI 1994), Port Ludlow, Washington (TARA 1998), Guadeloupe, French West Indies (SALSA 2002), Grundlsee, Austria (EDGE 2005), Banff, Alberta in Canada (EDGE 2009) the latest International Workshop on Enhanced Data Generated with Electrons (EDGE 2013) was held at Sainte Maxime from 26 to 31 May 2013.

This workshop took place at an exciting time for the EELS community as aberration correctors are spreading worldwide, enabling atomically resolved spectroscopy, and the next generation of monochromators are starting to emerge, promising improvements in energy resolution of more than one, possibly two, orders of magnitude compared with that of "naked" sources, with prospects for novel spectroscopy at energies not presently attainable. Papers highlighting such technique development, related advances in instrumentation for analytical transmission electron microscopy, and applications of EELS to materials and life science research, were presented at this workshop. These Proceedings thus provide a valuable "snapshot" of the state-of-the-art in the field of advanced spatially resolved spectroscopy using fast electron beams.

The workshop opened with a presentation by Christian Colliex entitled "Surviving 50 years of EELS measurements." Through his personal vision and experience, Christian told us the story of EELS, exploring the technical and scientific developments in the field over the past 50 years, and tracking, through pictures and comments, important milestones and highlights from previous editions of the EELS workshops. This entertaining lecture also discussed EELS in the present day and prospects for the future, serving as a perfect introduction to the program of talks and posters scheduled in the workshop.

In the following days a total of 156 papers were given, including 16 invited lectures, 41 contributed talks, and 99 posters, presented by an impressive mixture of young scientists and world-leading international experts.

A number of new and exciting ideas emerged during the workshop. For example, envisioning the future of monochromated EELS, some presenters focussed on the possibility to probe phonons or molecular vibrational modes (or even magnons) in the 1-100 meV range, and to access time-resolved spectroscopy. In nanooptics, many presentations highlighted the measurement of surface plasmon modes and associated spatial and temporal coherence effects in metallic nanostructures and recent advances in cathodoluminescence and its use in understanding quantum optics. Several presentations were also devoted to (soft and hard) X-ray spectroscopies and to "alternative" spectroscopy modes making use of electron vortex beams.

An impressive number of contributions addressed a wide range of materials science issues. Results were shown illustrating recent progress in the study of nanomaterials and nanostructures, including various types of two-dimensional materials, oxide heterostructures for various technological applications, nanostructured materials for batteries, and heterogeneous catalysts; the last example also being at the core of exciting and novel in situ experiments. Many contributions provided a clear demonstration that atomically resolved spectroscopy experiments, using both energy-dispersive X-ray spectroscopy and EELS, are now almost "routine" (although discussions clearly showed that there is still much to be done in developing theory and modeling for a quantitative interpretation of such spectroscopy data).

As the presentations in this workshop highlighted, EELS has certainly come a very long way since its inception and continues to have a very bright future ahead.

The workshop was supported financially by 16 sponsors, including all major manufacturers of instruments and accessories, three research institutions and two microscopy societies. 
The scientific activities were guided by an Organizing Committee comprised of Gianluigi Botton (Programme Chair), Peter Crozier (Programme Chair), Nigel Browning, Gerald Kotleiner, Ondrej Krivanek, Joachim Mayer, Paul Midgley, David Muller, Peter Nellist, Odile Stéphan, Masami Terauchi, and Maria Varela del Arco.

The success of this conference owes much to the Local Organizing Committee, led by Virigine Serin and Bénédicte Warot Fonrose, with the support of the French Microscopy Society. The workshop format followed that of previous meetings with the mornings and evenings devoted to talks and formal discussion, thus allowing moments in the afternoon for relaxation and contemplation in the midst of the fevered scientific discussion. The wet boat trip in the bays of Saint-Tropez and Pampelonne and the workshop party on the Sainte Maxime beach were certainly memorable moments!

A meeting with such an exciting program and so many stimulating discussions deserves a sequel and it was a unanimous decision that the workshops should continue on a 4 -year basis. The next workshop in 2017 will be organized by K. Kimoto and his Japanese colleagues in a location to be decided. We are looking forward to it!

Odile Stéphan

Laboratoire de Physique des Solides

Bâtiment 510, Université Paris-Sud, 91405 Orsay Cedex, France

odile.stephan@u-psud.fr

Paul Midgley

Department of Materials Science and Metallurgy

University of Cambridge, 27 Charles Babbage Road, Cambridge, CB3 OFS, UK pam33@cam.ac.uk 